\title{
Kondo effect of single Co atoms adsorbed on $\mathrm{Pb} / \mathrm{Si}(111)$ nanoislands
}

\author{
Xieqiu Zhang, ${ }^{1,2}$ Aidi Zhao, ${ }^{2}$ Kedong Wang, ${ }^{1}$ and Xudong Xiao ${ }^{1, *}$ \\ ${ }^{1}$ Department of Physics, The Chinese University of Hong Kong, Hong Kong, People's Republic of China \\ ${ }^{2}$ Department of Physics, The Hong Kong University of Science and Technology, Hong Kong, People's Republic of China
}

(Received 28 January 2008; revised manuscript received 10 April 2008; published 17 July 2008)

\begin{abstract}
Using scanning tunneling spectroscopy, we have investigated the local electronic property change upon single $\mathrm{Co}$ atom adsorption on $\mathrm{Pb}$ nanoislands and/or films grown on a $\mathrm{Si}(111)-7 \times 7$ surface. The quantum well states formed on the clean $\mathrm{Pb}$ film were found to be locally destroyed by the adsorbed single Co atom. Moreover, the differential conductance $d I / d V$ curves, exhibiting an asymmetric line shape with a dip and a hump, respectively, below and above the Fermi level, demonstrated a Kondo effect from the adsorbed Co atom on the $\mathrm{Pb}$ film. The similar line shapes of the $d I / d V$ spectra and the similar Kondo temperatures for Co atoms adsorbed on $\mathrm{Pb}$ island areas of different $\mathrm{Pb}$ thicknesses further showed that the different densities of states at the Fermi level originated from the quantum well states do not play a significant role, possibly due to the destruction of the quantum well states upon the Co atom adsorption.
\end{abstract}

DOI: 10.1103/PhysRevB.78.035431

PACS number(s): 72.15.Qm, 72.10.Fk, 73.21.Fg, 68.37.Ef

\section{INTRODUCTION}

The nanostructures with a permanent magnetic moment may play a key role in future spinelectronic technologies. ${ }^{1}$ Many of these systems can be described by the Kondo problem. ${ }^{2}$ The first observation of Kondo effect is from the measurement of resistivity of nonmagnetic metal with a small amount of magnetic impurities, where the resistivity anomalously increases at temperatures lower than the socalled Kondo temperature $\left(T_{K}\right)$. The explanation of Kondo effect involves the interaction between the magnetic impurities and the conduction electrons of the host metal. This interaction leads to a magnetic-moment screening effect by the spins of conduction electrons around the magnetic impurity and thus results in the anomalous resistivity at the temperatures lower than $T_{K}$.

The understanding of the physics in single magnetic atom adsorption on metallic surface is a fundamental basis to the possible applications of magnetic nanostructures. Combining the scanning tunneling microscopy (STM) and the scanning tunneling spectroscopy (STS), both the morphologic structure and the electronic structure can be probed for a single magnetic atom adsorbed on metal surfaces. The spectroscopic behavior of surface Kondo effect has been studied theoretically ${ }^{2-4}$ and experimentally. ${ }^{5-10}$ The experimental observations of the surface Kondo effect include $\mathrm{Mn} / \mathrm{Nb}(110){ }^{5}$ $\mathrm{Co} / \mathrm{Au}(111),{ }^{6} \mathrm{Ce} / \mathrm{Ag}(111),{ }^{7} \mathrm{Mn} / \mathrm{NiAl}(110),{ }^{8} \mathrm{Co} / \mathrm{Ag}(111)$, and $\mathrm{Co} / \mathrm{Cu}(111),{ }^{9}, 10$ just to name a few examples. Since the Kondo effect involves the spin-flip processes of the conduction electrons of the surrounding host nonmagnetic metal, naturally, one might ask what kind of role the density of states (DOS) near the Fermi level $\left(E_{F}\right)$ play in the Kondo effect.

Thin metallic films possessing quantum confinement effect can provide a way to tune the density of states near the Fermi level. ${ }^{11}$ For example, $\mathrm{Pb}$ film grown on semiconductor substrate shows quantum well states (QWSs), resulted from the electron confinement in the direction perpendicular to the film. ${ }^{11,12}$ In the presence of QWS, DOS at the Fermi level depends on the relative positions of QWSs from $E_{F}$, which is in turn determined by the thickness of the metal films. For $\mathrm{Pb}$ film or island, the increase in the film thickness leads to an oscillation of DOS at $E_{F}$, which has been demonstrated to induce an oscillation in the morphological structure, surface energy, thermal stability, and superconductivity. ${ }^{13-21}$ A very recent work has demonstrated that the Kondo temperature of a single manganese phthalocyanine $(\mathrm{MnPc})$ molecule adsorbed on $\mathrm{Pb}$ films oscillates with the thickness of the films. ${ }^{22}$ So far, the Kondo effect of a single magnetic atom on thin $\mathrm{Pb}$ films with different thicknesses has not yet been reported.

In this paper, we report our findings on the electronic property changes induced by a single magnetic Co atom adsorbed on the atomic flat surface of a $\mathrm{Pb}$ nanoisland grown on $\mathrm{Si}(111)-7 \times 7$. The QWSs were observed on the bare $\mathrm{Pb}$ surface area but disappeared on the adsorbed single Co atoms. The differential conductance spectra taken over single Co atoms showed asymmetric line shapes near the Fermi level, indicating the effect of Kondo resonance. The Kondo resonances on Co atoms adsorbed on surface areas of different $\mathrm{Pb}$ film thicknesses exhibited negligible difference, in contrast to the significantly different DOSs at the Fermi level on the bare $\mathrm{Pb}$ terraces. This insensitivity to the DOS at the Fermi level is consistent with our observation of the suppression of QWSs. Both effects indicate a strong interaction between the $\mathrm{Co}$ atom and the $\mathrm{Pb}$ surface, rendering a separate consideration of the QWSs of the $\mathrm{Pb}$ film and a perturbative picture of Co adsorption invalid.

\section{EXPERIMENT}

We used silicon wafer as the substrate. It was cut to a proper size followed by ultrasonic cleaning in water and acetone. After transfer into the ultrahigh vacuum, the wafer was first degassed at $600 \mathrm{~K}$ for $24 \mathrm{~h}$. The clean $\mathrm{Si}(111)-7 \times 7$ surface was prepared by a standard procedure: ${ }^{23}$ flashing it to $1520 \mathrm{~K}$ a number of times until the Si wafer could be held at $1520 \mathrm{~K}$ for 1 min under a pressure of $<1 \times 10^{-9}$ torr and cooling it quickly down to $1220 \mathrm{~K}$ and then slowly down to room temperature at a rate of $2 \mathrm{~K} / \mathrm{s}$. 

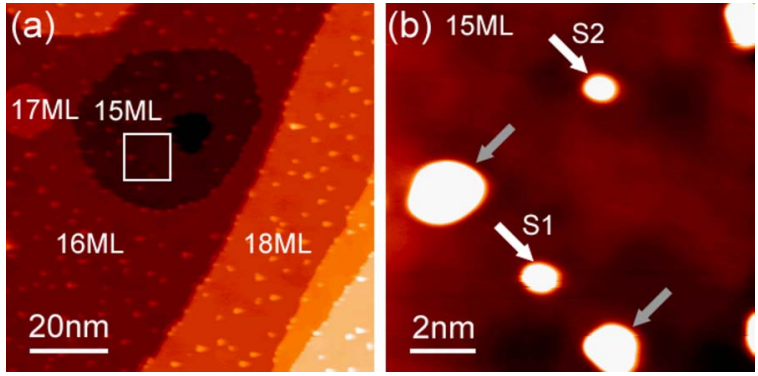

FIG. 1. (Color online) (a) A topographic STM image $\left(100 \times 100 \mathrm{~nm}^{2}\right)$ of a $\mathrm{Pb}$ island with $\mathrm{Co}$ atoms deposited on it. The image is taken at a bias of $+1 \mathrm{~V}$ and a tunnel current of $0.15 \mathrm{nA}$. (b) A close-up STM images $\left(15 \times 15 \mathrm{~nm}^{2}\right)$ of the square area in (a), taken at $+1 \mathrm{~V}$ and $0.15 \mathrm{nA}$. The sample temperature is $5 \mathrm{~K}$.

$\mathrm{Pb}$ was deposited onto the clean $\mathrm{Si}(111)-7 \times 7$ surface at a rate of 0.01 monolayer (ML)/s from an electron-beam evaporator. The $\mathrm{Pb}$ source was placed in a molybdenum crucible $\sim 20 \mathrm{~cm}$ away from the substrate surface and was thoroughly degassed before deposition. The $\mathrm{Si}(111)-7 \times 7$ substrate was kept at $170 \mathrm{~K}$ during deposition and the as-grown sample was annealed at room temperature for $1 \mathrm{~h}$. The formed $\mathrm{Pb}$ islands usually had a flat top or had a volcanoshaped top with a central hole surrounded by terraces. The latter kind of island could already provide a $\mathrm{Pb}$ film with a various thickness that is suitable for the thickness dependence studies. On top of the $\mathrm{Pb}$ island, Co atoms were evaporated from an electron-beam evaporator with a short Co rod as the source. Here, the sample was kept at room temperature without further annealing.

The measurements were carried out in the Omicron lowtemperature STM operated in ultrahigh vacuum with a base pressure of $7 \times 10^{-11}$ torr. The STM tip was a chemically etched Pt/Ir tip, which, prior to its first use, was cleaned by $\mathrm{Ar}^{+}$sputtering. All the measurements were performed at a temperature of $5 \mathrm{~K}$. We used the STM imaging mode to characterize the morphology of the sample surface and the STS mode of current $(I)$ versus bias voltage $(V)$ spectroscopy to measure the local electronic property. The differential conductance $d I / d V$ curves were numerically obtained from the tunneling $I-V$ spectra, which were smoothed using fast Fourier transformation method to filter out the high-frequency noise first. This smoothing procedure may lead to a broadening by about $15 \%$ in energy width for the relevant features reported in this paper.

\section{RESULTS AND DISCUSSION}

Figure 1 (a) shows a $100 \times 100 \mathrm{~nm}^{2}$ STM topographic image of a $\mathrm{Pb}$ island deposited with $\mathrm{Co}$ atoms. Because of the volcano-shaped top surface, this $\mathrm{Pb}$ island consists of terraces with a height ranging from 14 to $20 \mathrm{ML}$ with respect to the $\mathrm{Pb}$ wetting layer. The height difference between two adjacent terraces is of 1 or $2 \mathrm{ML}$. Before the Co atoms are deposited, the surface of the terrace is atomically flat with a corrugation smaller than $0.05 \mathrm{~nm}$. As shown in the large area STM image and in the close-up STM image in Fig. 1(b), the deposited Co atoms appear as small protrusions with typical
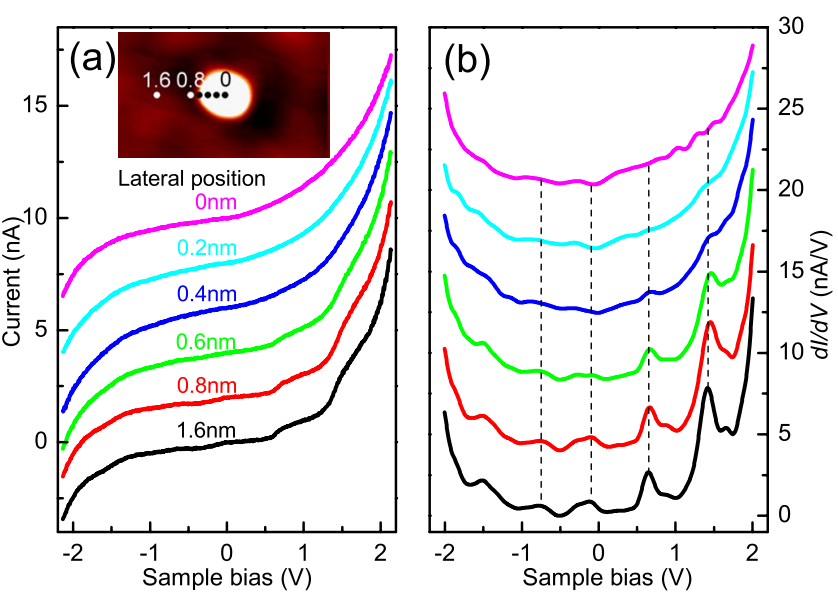

FIG. 2. (Color online) (a) The $I-V$ spectra and (b) the corresponding differential conductance $d I / d V$ spectra at different positions along a line near an adsorbed $\mathrm{Co}$ atom on the $\mathrm{Pb}$ island surface. The tip height is set by a bias of $+1.5 \mathrm{~V}$ and a tunnel current of $3 \mathrm{nA}$. The quantum well states appear as peaks in the $d I / d V$ spectra. As the STM tip moves toward the center of the Co atom, the quantum well peaks become vanished. The inset in (a) shows the positions at which the $I-V$ curves are taken.

lateral sizes from 1 to $3 \mathrm{~nm}$ and typical heights from 0.2 to $0.4 \mathrm{~nm}$. At this coverage, the adsorbed Co atoms can stay in single atom or small cluster form. Here, we regard the smallest protrusion features among all the adsorption structures as the single Co atoms. As labeled by $\mathrm{S} 1$ and $\mathrm{S} 2$ with white arrows in Fig. 1(b), these protrusions are quite circular in shape with a height of $0.2 \mathrm{~nm}$, comparable with the diameter of a Co atom. The round boundary of these features, in contrast to that of the larger protrusions labeled by gray arrows in Fig. 1(b), further supports the assignment of S1 and S2 to be single Co atoms. Due to the tip size convolution, the lateral size of such features is on the order of $1 \mathrm{~nm}$, larger than a single atom but comparable with the STM image size of a single $\mathrm{Mn}$ atom adsorbed on single-crystal $\mathrm{Nb}$ substrate ${ }^{5}$ in a different system. In this paper, we focus on the investigation of the electronic properties of single Co atom adsorption on $\mathrm{Pb}$ islands and will not discuss the results on $\mathrm{Co}$ clusters.

We have measured a series of $I-V$ spectra at different lateral positions along a line with respect to a Co atom $(\mathrm{S} 1)$ for energies ranging from -2 to $+2 \mathrm{eV}$. The corresponding $I-V$ spectra and numerically differentiated $d I / d V$ curves are shown in Figs. 2(a) and 2(b), respectively, where vertically shifts are made for clarity. The inset in Fig. 2(a) shows the positions at which the spectra were taken with respect to the center of protrusion $\mathrm{S} 1$. The set of peaks in the $d I / d V$ spectrum on bare $\mathrm{Pb}$ surface [the bottom curve in Fig. 2(b)] is due to the QWSs formed in the Pb thin films. ${ }^{11,12}$ The positions of the QWS peaks are indicated by the vertical dashed lines. These QWS peaks remain intact at the edge of the protrusion (labeled $0.8 \mathrm{~nm}$ ) and start to diminish monotonically when the STM tip continues to move toward the center of the protrusion. At around $0.2 \mathrm{~nm}$ away from the protrusion center, all the QWS peaks are practically vanished with no signature of the quantum well states remaining. 


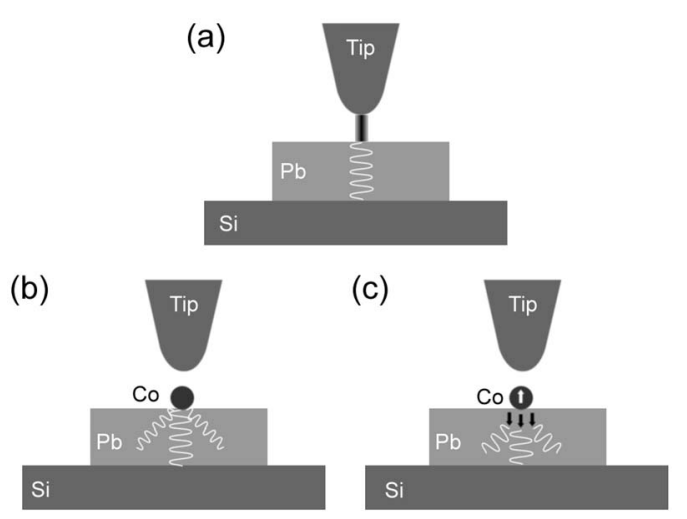

FIG. 3. (a) A sketch showing the quantum well states in the $\mathrm{Pb}$ island and the detection by an STM tip. (b) A sketch showing the scattering of the quantum well state electrons by an atom with weak interaction. (c) A sketch showing the Kondo effect, namely, the conduction electron cloud near a magnetic impurity, forms a shell structure with opposite spin orientation in the adjacent regions. A strong interaction destroys the quantum well states.

To understand the suppression of QWSs by a single Co atom, we have drawn schematics for the tunneling processes probed by an STM tip on bare Pb surface in Fig. 3(a) and on Co atom adsorbed on the Pb film in Figs. 3(b) and 3(c). In Fig. 3(a), the quantization of electronic wave in the perpendicular direction leads to a formation of electronic standing wave (corresponding to a QWS) inside the $\mathrm{Pb}$ island, similar to a standing light wave in a Fabry-Pérot optical interferometer. The tunneling current between the $\mathrm{Pb}$ film surface and the STM tip is determined by the probability of electrons tunneling through the classical forbidden vacuum area. In the case of Fig. 3(b), where the Co atom is assumed to weakly interact with the film surface, the quantization condition in the perpendicular direction remains intact inside the $\mathrm{Pb}$ island and the density of states of the QWSs remains unchanged. Due to the energy-level mismatch between the single $\mathrm{Co}$ atom and the $\mathrm{Pb}$ islands, the $\mathrm{Co}$ atom behaves like an atomic scattering center, reducing the electrons from QWSs to tunnel into the STM tip or the electrons to tunnel from the STM tip into the empty QWSs. In this case, the single Co atom just behaves like an attenuator to reduce the intensity of the tunneling current at all electronic energies including those of the QWS peaks. Indeed, such a behavior was found for $\mathrm{MnPc}$ molecule adsorbed on $\mathrm{Pb}$ films, as reported in Ref. 22. However, as judged from the $d I / d V$ curves in Fig. 2(b), in the tunneling spectrum on the Co atom, while an overall smooth varying intensity exists, the peak features are completely washed out. This indicates that, instead of a simple attenuation by the Co atom adsorption, the QWSs have been destroyed locally. Our results shown in Fig. 2(b) imply that the interaction between the adsorbed Co atom and the $\mathrm{Pb}$ surface is so strong that the Co atom cannot be regarded as a perturbation on the $\mathrm{Pb}$ film. Instead, the adsorbed Co atom must be considered to alter the boundary conditions for the electrons within the Pb film. In Fig. 3(c), we speculate one possible interpretation. In order to screen out the magnetic-dipole moment of the Co atom, the electrons surrounding the magnetic impurity will form electron cloud

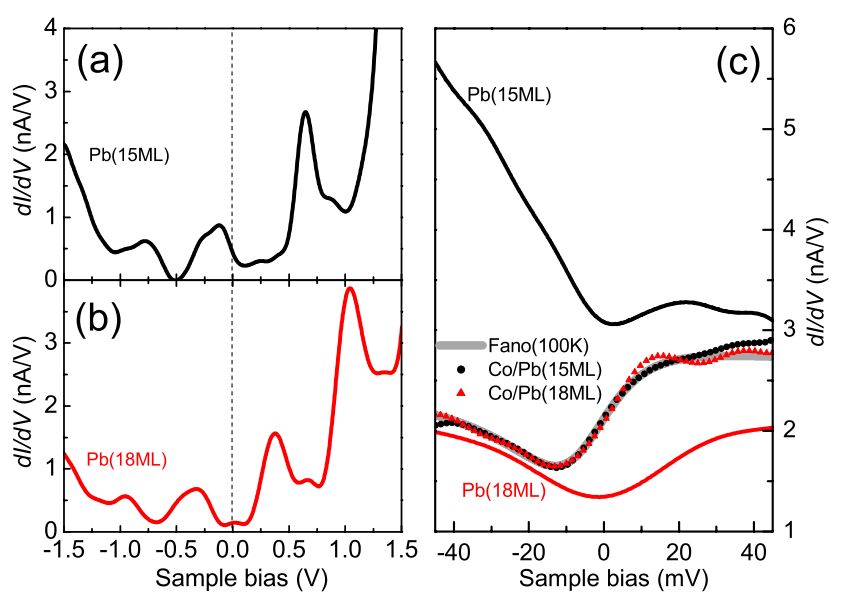

FIG. 4. (Color online) (a) The differential conductance $d I / d V$ spectra on a terrace of $15 \mathrm{ML} \mathrm{Pb}$ thickness. The tip height is set by a bias of $+1.5 \mathrm{~V}$ and a tunnel current of $3 \mathrm{nA}$. (b) Similar to (a) but on a terrace of $18 \mathrm{ML} \mathrm{Pb}$ thickness. (c) The $d I / d V$ spectra in a smaller energy range on bare $\mathrm{Pb}$ terraces of 15 and $18 \mathrm{ML}$ thick and on the single Co atoms adsorbed on terraces of 15 and $18 \mathrm{ML} \mathrm{Pb}$ thick. The tip height is set by a bias of $0.1 \mathrm{~V}$ and a tunnel current of $0.3 \mathrm{nA}$. A fitting line with Fano line shape to the $d I / d V$ spectra on Co atoms with a Kondo temperature $T_{K} \sim 100 \mathrm{~K}$ is also shown in a thick gray line.

possessing the opposite spins. This collective electron-spin fluctuation around the single magnetic impurities may act as scattering centers and amplify the effect from a single Co atom to modify the boundary conditions of the $\mathrm{Pb}$ top surface. Thus, the QWSs can no longer form near the site of the Co atom. We estimated that a scattering center with an energy barrier of $\sim 4 \mathrm{eV}$ and a barrier width of $\sim 1 \mathrm{~nm}$ is sufficient to backscatter the QWS electrons. At this stage, we cannot pin down the mechanism for the elimination of the QWSs and further work is required. Since the Kondo resonance in the low-temperature region $T<T_{k}$ is a pure potential scatterer in the Fermi-liquid theory, ${ }^{4,24}$ this leads us to consider the surface Kondo effect induced by single Co atoms.

To investigate the Kondo effect and its dependence on DOS at the Fermi level, we have measured the local electronic spectroscopy near the Fermi level both on the Co atom and off the $\mathrm{Co}$ atom (on the bare $\mathrm{Pb}$ surface) for two terraces with different $\mathrm{Pb}$ thicknesses. As shown in Figs. 4(a) and 4(b), the Fermi level for the $15 \mathrm{ML}$ terrace lies on the shoulder of a QWS peak centered at $-0.11 \mathrm{eV}$, resulting in an enhanced DOS at the Fermi level. In contrast, the Fermi level for the $18 \mathrm{ML}$ terrace lies at the valley between two QWSs at -0.32 and $+0.38 \mathrm{eV}$, providing a low DOS at the Fermi level. The ratio of the DOS at Fermi level for the 15 and $18 \mathrm{ML}$ terraces is around 3-4. The $d I / d V$ curves on the 15 and 18 ML bare Pb terraces show very different shapes, as seen especially clear in Fig. 4(c) within a smaller energy range. The dip at the Fermi level in the $d I / d V$ spectra of the bare $\mathrm{Pb}$ surface is not due to the Co adsorption since it was observed on clean $\mathrm{Pb}$ surface even before Co deposition. The nature of this dip is beyond the present paper and will be discussed elsewhere. ${ }^{25}$ 
Here, we focus on the comparison of the $d I / d V$ spectra on the $\mathrm{Co}$ atom with those on the bare $\mathrm{Pb}$ surface. In addition to the $d I / d V$ spectra taken on the bare $\mathrm{Pb}$ surfaces, Fig. 4(c) shows the $d I / d V$ curves taken on single Co atoms adsorbed on terraces of 15 and $18 \mathrm{ML} \mathrm{Pb}$ thick. As shown by the dotted line, the $d I / d V$ curve taken on the Co atom adsorbed on the $15 \mathrm{ML} \mathrm{Pb}$ terrace (site $\mathrm{S} 1$ ) shows a much reduced intensity as compared to the spectrum on the corresponding bare $\mathrm{Pb}$ surface, in particular for energy below the Fermi level. This indicates that by adsorption of a Co atom, some of the bare film electronic states in the QWS peaks are now removed and redistributed to the QWS valleys. These distinctive spectra are consistent with our observation of the suppression of QWSs by the single Co atom adsorption. Similarly, on the $18 \mathrm{ML} \mathrm{Pb}$ terrace, adsorption of Co destroys the QWSs, but, this time, the intensity of the $d I / d V$ spectrum is increased, indicating that some of the forbidden electronic states caused by the quantum interference in the bare film are now allowed. In another words, the decreased number of electronic states near Fermi level (redistributed to QWS peaks nearby) by quantum interference in the bare 18 ML film is now returned upon Co adsorption. Surprisingly, the spectra on the Co atom adsorbed on both 15 and $18 \mathrm{ML}$ high terraces, shown by the dotted line and triangle line (data points in triangles), respectively, are practically the same, in strong contrast to the large DOS difference caused by QWSs in the same energy region for the bare films. This again supports that the QWSs are completely suppressed. The asymmetric line shape with a dip below the Fermi level and a hump above the Fermi level is a signature of Kondo effect, as reported for a number of other magnetic atoms adsorbed on metallic surfaces. ${ }^{6,7,9}$ Such a Kondo line-shape feature was not clearly shown in Fig. 2(b) due to the lower energy resolution and lower differential conductance used there. Using the Fano line-shape formula, 3,4,9

$$
\frac{d I}{d V} \propto \frac{(q+\widetilde{\varepsilon})^{2}}{1+\widetilde{\varepsilon}^{2}},
$$

where $\tilde{\varepsilon}=\left(e V-\varepsilon_{K}\right) / \Gamma, e$ is the electron charge, $V$ is the bias voltage, $q$ characterizes the line shape, $\varepsilon_{K}$ is the energy position of the resonance with respect to the Fermi level, and $\Gamma$ determines the half width of the Kondo resonance and the Kondo temperature $\left(\Gamma=2 k_{B} T_{K}\right)$; we have fitted the $d I / d V$ curves on the single Co atoms on the 15 and $18 \mathrm{ML} \mathrm{Pb}$ terraces. We find that $T_{K} \sim 100 \pm 10 \mathrm{~K}, q \sim 0.5 \pm 0.05$, and $\varepsilon_{K} \sim-5 \mathrm{meV}$ can fit the data quite well for both cases [thick gray line in Fig. 4(c)]. The Kondo temperature obtained here may have been overestimated by $\sim 15 \%$ due to the smooth- ing procedure for both cases since the same smoothing procedure is used. Within the experimental uncertainty, the Kondo resonance of $\mathrm{Co}$ atom on both 15 and $18 \mathrm{ML} \mathrm{Pb}$ terraces clearly does not exhibit any noticeable thickness dependence.

Our observation on the Kondo effect of Co atom is different from the recent report for $\mathrm{MnPc}$ on the same metal host, $\mathrm{Pb}$ films, ${ }^{22}$ for which a clear dependence of the Kondo resonance on the $\mathrm{Pb}$ film thickness was observed. The line shapes of the Kondo resonance for $\mathrm{Co}$ and $\mathrm{MnPc}$ are also very different and are described by Fano line shape for Co but by Doniach-Sunjic line shape for MnPc. We speculated that the different behaviors are due to the strong interaction between the single $\mathrm{Co}$ atom with $\mathrm{Pb}$ surface, in contrast to the weak interaction between $\mathrm{MnPc}$ and the $\mathrm{Pb}$ surfaces. In the case of Co atom, it has two electrons in the $s$ orbital and seven electrons in the $d$ orbital. All these electrons can interact with the $\mathrm{Pb}$ surface. However, for MnPc molecule the $s$ orbital in $\mathrm{Mn}^{2+}$ is empty and the interaction between $\mathrm{Mn}^{2+}$ and the $\mathrm{Pb}$ surface is mostly from the $d z^{2}$ orbital. ${ }^{22}$ After forming bonds with the Pc ligands, it is expected that the interaction from the $d$-orbital electrons with the $\mathrm{Pb}$ surface is significantly weaker. This speculation is supported by the contrast between our observation of QWS suppression by Co adsorption and their observation of the intactness of QWSs by $\mathrm{MnPc}$ molecule adsorption. ${ }^{22}$

\section{CONCLUSIONS}

In summary, we have investigated the electronic structure changes induced by adsorption of single Co atoms on the $\mathrm{Pb}$ island and/or film grown on $\mathrm{Si}(111)-7 \times 7$. The quantum well states observed on the clean $\mathrm{Pb}$ island were found to be destroyed by the single Co atom adsorption. The spectra near the Fermi level taken on the single Co atoms have shown a different line shape from those on the bare $\mathrm{Pb}$ surface, indicating the existence of a Kondo resonance. The large difference in the DOS at Fermi level on the clean terraces with different $\mathrm{Pb}$ thicknesses induced by the QWSs does not lead to a significant difference in the Kondo effect of single Co atoms, supporting the notion of the QWS suppression under Co atom. Our results indicate that the interaction strength between an adsorbate and the $\mathrm{Pb}$ surface is critical for affecting the robustness of the QWSs.

\section{ACKNOWLEDGMENTS}

This work was supported by the Research Grants Council of Hong Kong (Grant No. CUHK603806).

\footnotetext{
*Corresponding author; xdxiao@phy.cuhk.edu.hk

${ }^{1}$ P. Gambardella, S. Rusponi, M. Veronese, S. S. Dhesi, C. Grazioli, A. Dallmeyer, I. Cabria, R. Zeller, P. H. Dederichs, K. Kern, C. Carbone, and H. Brune, Science 300, 1130 (2003).

${ }^{2}$ A. C. Hewson, The Kondo Problem to Heavy Fermions (Cambridge University Press, Cambridge, 1993).
}

${ }^{3}$ U. Fano, Phys. Rev. 124, 1866 (1961).

${ }^{4}$ O. Újsághy, J. Kroha, L. Szunyogh, and A. Zawadowski, Phys. Rev. Lett. 85, 2557 (2000).

${ }^{5}$ A. Yazdani, B. A. Jones, C. P. Lutz, M. F. Crommie, and D. M. Eigler, Science 275, 1767 (1997).

${ }^{6}$ V. Madhavan, W. Chen, T. Jamneala, M. F. Crommie, and N. S. 
Wingreen, Science 280, 567 (1998).

${ }^{7}$ J. Li, W.-D. Schneider, R. Berndt, and B. Delley, Phys. Rev. Lett. 80, 2893 (1998).

${ }^{8}$ A. J. Heinrich, J. A. Gupta, C. P. Lutz, and D. M. Eigler, Science 306, 466 (2004).

${ }^{9}$ P. Wahl, L. Diekhöner, M. A. Schneider, L. Vitali, G. Wittich, and K. Kern, Phys. Rev. Lett. 93, 176603 (2004).

${ }^{10}$ N. Quaas, M. Wenderoth, A. Weismann, R. G. Ulbrich, and K. Schönhammer, Phys. Rev. B 69, 201103(R) (2004).

${ }^{11}$ T.-C. Chiang, Surf. Sci. Rep. 39, 181 (2000).

${ }^{12}$ I. B. Altfeder, K. A. Matveev, and D. M. Chen, Phys. Rev. Lett. 78, 2815 (1997).

${ }^{13}$ K. Budde, E. Abram, V. Yeh, and M. C. Tringides, Phys. Rev. B 61, R10602 (2000).

${ }^{14}$ W. B. Su, S. H. Chang, W. B. Jian, C. S. Chang, L. J. Chen, and Tien T. Tsong, Phys. Rev. Lett. 86, 5116 (2001).

${ }^{15}$ A. E. Meyerovich and D. Chen, Phys. Rev. B 66, 235306 (2002).

${ }^{16}$ C. M. Wei and M. Y. Chou, Phys. Rev. B 66, 233408 (2002).
${ }^{17}$ P. Czoschke, H. Hong, L. Basile, and T.-C. Chiang, Phys. Rev. Lett. 91, 226801 (2003).

${ }^{18}$ P. Czoschke, H. Hong, L. Basile, and T.-C. Chiang, Phys. Rev. Lett. 93, 036103 (2004).

${ }^{19}$ M. H. Upton, C. M. Wei, M. Y. Chou, T. Miller, and T.-C. Chiang, Phys. Rev. Lett. 93, 026802 (2004).

${ }^{20}$ Y. Guo, Y.-F. Zhang, X.-Y. Bao, T.-Z. Han, Z. Tang, L.-X. Zhang, W.-G. Zhu, E. G. Wang, Q. Niu, Z. Q. Qiu, J.-F. Jia, Z.-X. Zhao, and Q.-K. Xue, Science 306, 1915 (2004).

${ }^{21}$ Y.-F. Zhang, J.-F. Jia, T.-Z. Han, Z. Tang, Q.-T. Shen, Y. Guo, Z. Q. Qiu, and Q.-K. Xue, Phys. Rev. Lett. 95, 096802 (2005).

${ }^{22}$ Y.-S. Fu, S.-H. Ji, X. Chen, X.-C. Ma, R. Wu, C.-C. Wang, W.-H. Duan, X.-H. Qiu, B. Sun, P. Zhang, J.-F. Jia, and Q.-K. Xue, Phys. Rev. Lett. 99, 256601 (2007).

${ }^{23}$ B. S. Swartzentruber, Y.-W. Mo, M. B. Webb, and M. G. Lagally, J. Vac. Sci. Technol. A 7, 2901 (1989).

${ }^{24}$ P. Nozières, J. Low Temp. Phys. 17, 31 (1974).

${ }^{25}$ K. Wang, X. Zhang, M. M. T. Loy, and X. Xiao (unpublished). 